\title{
Tartaric Acid
}

National Cancer Institute

\section{Source}

National Cancer Institute. Tartaric Acid. NCI Thesaurus. Code C47744.

A white crystalline dicarboxylic acid found in many plants, particularly tamarinds and grapes. Tartaric acid is used to generate carbon dioxide through interaction with sodium bicarbonate following oral administration. Carbon dioxide extends the stomach and provides a negative contrast medium during double contrast radiography. In high doses, this agent acts as a muscle toxin by inhibiting the production of malic acid, which could cause paralysis and maybe death. 